Schweiz. Z. Path. Bakt. 1958;21:I-VIII

\title{
Contents, Vol. 21, 1958
}

\section{H. BERGSTRAND, Stockholm}

R. BIELING, Wien

H. CHIARI, Wien

M. DJURISIC, Beograd

J. FIRKET, Liège

E. GRASSET, Geneve $\dagger$

C. HALLAUER, Bern

E. LETTERER, Tubingen

EDITORES

A.LINDAU, Lund $\dagger$

E. G. NAUCK, Hamburg

J. L. NICOD, Lausanne

CH. OBERLING, Paris

A. L. OLITZKI, Jerusalem

F. C. ROULET, Basel

E. RUTISHAUSER, Geneve H. SMETANÀ, Washington

J. TOMCSIK, Basel

R. TULASNE, Strasbourg

E. UEHLINGER, Zurich

H. UNGAR, Jerusalem

B. WALTHARD, Bern

M. WELSCH, Liège

A. WERTHEMANN, Basel

K. C. WINKLER, Utrecht

REDACTORES A. v. ALBERTINI, Zurich A. GRLMBACH, Zurich H. MOOSER, Zurich Vol. 21

1958

BASEL (Schweiz) S. KARGER NEW YORK

Index

Ahrens, C.B. F.W.: vide Fuchs, G. H. P.

Albertini, A. von : Studien zur Karzinogenese I. Die menschlichen Präkanzerosen . . 688

Albertini, A. von: Studien zur Karzinogenese II. Experimentelles Hautkarzinom mit

Methylcholanthren 773

Andrejevic, Mm.: Les caractéristiques de Гulcère gastro-duodénal en Yougoslavie . . 213

Andrejevic, Mih. et Ivankovic, D.:

La possibilité d'une medication combinée dans la maladie ulcéreuse

et les nouveaux medicaments 568

Areán, V. M.: The Natural History of Atherosclerosis in Puerto Rico

Avery Jones, F.: vide Doll, R. 
Balestra, V.: Considerations statistiques sur la mortalité par ulcère gastro-duo dénal dans la ville de Genes 223

Balestra, V.: La diffusion de $\Gamma$ ulcère gastro-duodénal en Italie 449

Balestra, V.: La frequence de la mort par tuberculose parmi les sujets opérés pour ulcère gastro-duodénal 559

Balestra, V. et Mattioli, F.:

Les groupes sanguins et $\Gamma$ ulcère gastro-duodénal 331

Baló, J.: $\quad$ Système nerveux et ulcère peptique 561

Baló, J., Besznyák, I. und Kendrey, G.:

Die Rolle der Virushepatitis für die Entstehung der Leberzirrhose . 1064

Banic, S.: Isolation by the Replica Plating Technique of the Mutants of Staph.

aureus, Partially Resistant to Low Concentrations of Penicillin. . . 54

Baumann-Grace, J.B. und Tomcsik, J.:

Elektronenmikroskopische Untersuchung der komplexen Kapsel-

struktur bei B. megaterium 906

Baumann-Grace, J.B.: vide Tomcsik, J.

Beaune, A.: vide Dupuy, R.

Benda, R., Carvaillo, R., Miravet, L. F. et Gartenlaub, G.:

Application du «test de protection» à $\Gamma$ étude du serum sanguin

dans les ulcères gastro-duodénaux 338

Bernier, J. J.: vide Lambling, A.

Bertrand, J.: vide Lambling, A.

Bertschmann, M.: vide Regamey, R. H.

Besznyák, I.: vide Baló, J.

Bolle, A. et Kellenberger, E.:

Etude de Taction du laurylsulfate de sodium sur E. coli $\quad 714$

Bonfils, S., Rene, L., Richir, Cl., Hardouin, J.P. et Lambling, A.:

Etude histologique du rein au cours de $\Gamma$ ulcère gastrique et duode

nal. (Biopsies per-opératoires) 547

Bras, G. and Jelliffe, D.B.:

Observations on the Geographical Pathology of Cirrhosis of the Liver

in Infants and Children 599

Brodhage, H.: Vergleichende TJntersuchungen an 20 Seren mit der Agglutinations-

reaktion auf Brucellose in verschiedenen Instituten 1023

Bruhin, H.: vide Tirunarayanan, M. 0.

Büchner, F.: Die Pathogenese der Gastro-Duodenal-Geschwüre 388

Cabanne, F.: vide Letac, R.

Cagianut, B.: Steroide und Wachstum. Zur Wirkung der Nor-Steroide

Carvaillo, R.: vide Benda, R.

Concepción, C.: vide Morera, A.

Conte, M. et Julien, Cl.:

L'ulcère gastro-duodénal chez les Nord-Africains immigrés travail-

lant dans la region parisienne 238

Copelman, L. S.: La geographic des ulcères gastro-duodénaux et $\Gamma$ expansion du goitre

endémique. Influence du système neuro-hormonal. La teneur de Гeau

en calcium et le goitre endémique $\quad 562$ 
Cossel, L.: Nierenbefunde beim Meerschweinchèn bei Klossiellen-Infektion(Klossiella cobayae). (Zur Kenntnis der speziellen Pathologie der Versuchstiere) 62

Dabrowski, L.: vide Slopek, St.

Debray, Ch. et Hardouin, J. P.:

Etude biologique de Pulcère gastro-duodénal 286

Debray, Ch.: vide Delarue, J.

Delarue, J.: Allocution 124

Delarue, J., Debray, Ch., Mignot, J. et Thomas, J.:

Etude de la vascularisation des ulcères gastriques 496

Demole, M. et Secrétan, Ph.:

Ulcère gastro-duodénal et tuberculose pulmonaire 553

Djurisic, M. und Drndarski, K.:

Über das Problem der sog. «Viable et non-viable »-Tuberkelbazillen

bei der experimentellen Tuberkulose der Meerschweinchèn, die mit

großen Einzeldosen von Streptomycin, Isoniazid und deren Kombi-

nationen behandelt wurden 115

Doll, R. and Avery Jones, F.:

Smoking and Peptic Ulcer 309

Drimmer-Herrnheiser, H. and Olitzki, A. L.:

Alcaligenes haemagglutinans: Factors Determining the Agglutinability

of the Red Blood Cells and the Mechanism of the Agglutination .. 93

Drndarski, K.: vide Djurisic, M.

Dubarry, J. J. et Pisot, Ch.:

Importance de Thérédité dans la pathogénie de la maladie ulcéreuse 314

Dungal, N. and Hansen, H.:

Peptic Ulcers in Iceland 225

Dupuy, R. et Beaune, A.:

Sur la formule protidique sérique de l'ulcère gastro-duodénal dans

divers groupes ethniques 333

Edgcomb, J.H.: On the Development of Peptic Ulcers in Patients Treated with Prednisone or Prednisolone $\quad 363$

Eser, S. et Velicangil, S.:

Le goitre endémique à Isparta (Turquie) et son étiologie 629

Fey, H.: Zum klinischen Begriff der «septischen» coliformen Mastitis. . . 926

Flamm, H. und Kovac, W.:

Die Pathogenese der pseudotuberkulösen Lymphadenitis ileocaecalis 1127

Fluder, Z.: vide Pakula, R.

Franchini, A. et Mattioli, G.:

Considerations étio-pathogéniques chez 1000 malades d'ulcère gastro-

duodénal (en s'arrêtant particulièrement sur les facteurs géographi-

ques) $\quad 460$

Franchini, A.: vide Mattioli, G.

Friedrich, L. et Havas, E.:

Le problème des ulcères en Hongrie 446

Fuchs,G.H.P. und Ahrens, C.B.F.W.: 
Neue zeit- und materialsparende Möglichkeiten bei der Herstellung des Basalmediums für den Nelson-Mayer-Test

Galindo, L.: vide Lichtenberg, F.

Gandara, L.: vide Mogena, H. G.

Gartenlaub, G.: vide Benda, R.

Gault, E. W.: vide Hadley, G. G.

Gross, F.: vide Kradolfer, F.

Hadley, G. G.: A Study of Peptic Ulcer as Found in South India

Hadley, G.G., Gault, E.W. and Job, C.K.:

Cardiovascular Disease in South India - Based on Postmortem Expe

rience 622

441233

Hansen, J. L.: Necropsy Statistics on Chronic Gastric and Duodenal Ulcer in Copen hagen During Forty Years: A Method to Calculate the Incidence in

the Living Population from Autopsy Figures

Hansen, H.: vide Dungal, N.

Hardouin, J. P.: Les Ulcères gastro-duodénaux chez les Nord-Africains Musulmans à

Paris

Hardouin, J.P.: vide Bonfils, S.

Hardouin, J. P.: vide Debray, Ch.

Havas, E.: vide Friedrich, L.

Henschen, $F \cdot$ : La pathologie géographique des ulcères gastroduodénaux en Suède . 209

Henschen, F.: La frequence des lesions peptiques dans un materiel d'autopsies de

Stockholm (selon les recherches du Dr Falconer) 437

Herzog, E.: Über Mißbildungen und ihre statistische Erfassung im Sektionsgut

des Pathologischen Institute der Universität Concepción (Chile) . . 572

Higginson, J. and Simson, I.:

Lesions of the Gastro-Intestinal Tract in the Non-White Population

of South Africa $\quad 577$

Hiltbold, B., Huber, L. und Regamey, R.A.:

Aktive Immunisierung gegen H. pertussis: Die Entwicklung der

humoralen Antikörper beim Kaninchen. II. Mitteilung 939

Huber, L.: vide Hiltbold, B.

Ivankovic, D.: vide Andrejevic, Mih.

Iverson L. Choriocarcinoma and Related Conditions in Southeast Asia .... 581

Jelliffe, D.B.: vide Bras, G.

Job, C. K.: vide Hadley, G. G.

Julien, $\mathrm{Cl}$.: vide Conte, $\mathrm{M}$.

Jusatz, H. J.: Medizinische Kartographie als wissenschaftliche Methode zur Erfor-

schung der Krankheitsvorkommen auf der Erde $\quad 618$

Kañtoch, M., Skurski, A. and Wieczorek, Z.:

In Vitro Blockade of Bacterial Phagocytosis of Leukocytes by Means

of Bacterial Viruses 1106

Kasich, A.M.: Hexocyclium Methosulfate, a New Anticholinergic Drug in Conventional and Long-Acting Forms: Its Effects on Gastric Secretion . . 354

Kellenberger, E.: vide Bolle, A. 
Keller, R,: Quantitative Untersuchungen zur Beziehung zwischen Mastzellveränderungen und Histaminausscheidung bei der Albinoratte $\quad 903$

Kendrey, G.: vide Baló, J.

Kétyi, I.: vide Rauss, K.

Köstlin, A.: vide Rauch, S.

Kolc, J.: vide Trnka, Z.

Konsanszky, A.: Veränderungen des osmotischen Druckes im Laufe der Fermenta

tion. I. Zusammenhänge zwischen der Veränderung des osmotischen

Druckes und der Vitamin B,-Produktion 659

Konsanszky, A.: Veränderungen des osmotischen Druckes im Laufe der Fermentation. II. Die

Veränderungen im Gehalt des Nährbodens an Kohlehy-draten, Eiweiß und organisch

gebundenem Stickstoff sowie die Ge-staltung des osmotischen Druckes während der

Fermentation ... 662

Konstam, P.G.: Peptic Ulcer in West Africa and India

KovÁcs, E.: vide Matkovics, B.

Kovac, W.: vide Flamm, H.

Kradolfer, F. und Gross, F.:

Die Ingestion von Erythrozyten durch E. histolytica 1014

Kradolfer, F. und Wyler, R.:

Unspezifische Resistenzfaktoren bei Virusinfektionen

Kradolfer, F.: vide Wyler, R.

Kucsko, L.: Das Verhalten der Ulcuskrankheit auf Grund der Sektionsprotokolle

des Wiener pathologisch-anatomischen Institutes in den letzten 100

Jahren 433

Kunz, Ch. : Untersuchungen mit fluoreszein-markierten Antikörperø an Hefen . 892

Kurokawa, T. and Masuda, H.:

Clinical Observations on Peptic Ulcer in Japan

Lambling, A. et Bernier, J. J.:

Physiologie de la secretion gastrique 132

Lambling, A. et Richir, $\mathrm{Cl}$.:

Les lesions histologiques de la muqueuse gastrique au cours de $\Gamma$ ulcère gastrique et duodenal.

Etude statistique à propos de 104 cas . . 318

Lambling, A., Bertrand, J. et Bernier, J. J.:

Les variations de la secretion chlorhydrique en fonction de la richesse cellulaire des glandes

fundiques au cours des ulcères gastro-duodénaux 325

Lambling, A.: vide Bonfils, S.

Letac, R., Mawupe Vovor, V. et Cabanne, F.:

De la rareté de la pathologie veineuse des membres inférieurs en

Afrique Noire 587

Lichtenbebg, F. and Galindo, L.:

Trends and Changes in Mortality Causes in Puerto Rico Based on

Autopsy Material between 1926-1953

Likar, M.: Some Observations on the Value of Virus Isolations and Serological

Tests for the Routine Diagnosis of Neurotropic Virus Infections -

Slovenian Experience 670

Lindenmann, J. und Vogel, A.: 
Die Färbung des Sendai-Virus

1008

Lisowski, J., Wieczorek, Z. and Skurski, A.:

Effect of Thiol Compounds on Phagocytosis 1096

Löffler, H.: Zur Hämagglutination durch Influenzavirus A-Asia 1002

Lopes de Faria, J.: Medianecrosis Aortae after Collapse in Rabbits 103

MacIver, J.E.: vide Went, L.N.

Málek, P.: vide Trnka, Z.

Mariani, P. L. et Viola, G.:

Observations anatomo-cliniques sur la maladie ulcéreuse $\quad 457$

Masuda, H.: vide Kurokawa, T.

Matkovics, B. and KovÁcs, E.:

A Simple Method for $\mathrm{rH}$ Measuring in Microbiological Processes . . 666

Mattioli, G. et Franchini, A.:

Etude clinique portant sur 1000 ulcéreux gastro-duodénaux opérés

à Bologne 467

Mattioli, G.: vide Franchini, A.

Mattioli, F.: vide Balestra, V.

Mawupe Vovor, V.: vide Letac, R.

May, J.M.: $\quad$ Report on the Geography of Peptic Ulcers 169

Meisel, H. und Rymkiewicz, D.:

Über die serologischen Eigenschaften der durch Enzymbehandlung

freipräparierten Bakteriensporen 866

Metzger, M.: Studies on the Heat-Labile Antigens in the Boyd-Group of Shigella

Bacilli 645

Metzger, M.: vide Slopek, St.

Michalowicz, M.: Sensibilité générale et individuelle aux processus électrologiques et électro-magnétiques naturels et artificiels $\quad 575$

Mignot, J.: vide Delarue, J.

Milgrom, F., Wicher, K. and Rogala, D.:

Effect of Intraembryonic Injections of B.C.G. on the Survival Time

in the Tuberculous Guinea-pig

89

Miravet, L. F.: vide Benda, R.

Mogena, H. G. et Gandara, L.:

Résultats lointains du traitement medical de $\Gamma$ Ulcère gastro-duodénal 565

Morera, A. et Concepción, C.:

L'influence du biotype sur $\Gamma$ apparition de la perforation des ulcères

gastro-duodénaux 499

Nicod, J.L.: $\quad$ Monstre double en 0 chez Thomme 682

Okabayashi, A.: The Significance of Allergy in the Development of Gastric Peptic

Ulcer 490

Olitzki, A. L.: vide Drimmer-Herrnheiser, H.

Pakula, R., Fluder, Z., Walczak, W. and Zakrzewski, K.:

Albumin as Sensitizing Factor in Transformation of Pneumococci to

Streptomycin Resistance 1119

Pa yet, M.: Aspect particulier des ulcères gastro-duodénaux en Afrique Française et à Madagascar $\quad 475$ 
Pijper, A.: Flagella, motility, and agglutination of Pseudomonas viscosa . . 707

Pisi, E.: vide Sotgiu, G.

Pisot, Ch.: vide Dubarry, J. J.

Pletscher, A.: vide Zbinden, G.

Pulvertaft, C. N.: The Incidence of Peptic Ulceration in York and Environs 220

Rauch, S. und Köstlin, A.:

Die optimale Wunddesinfektion $\quad 1$

Rauss, K. and Ketyi, I.:

Some Data Regarding the Mechanism of Indirect Bacterial Haemag-

glutination and Haemagglutination Inhibition

Regamey, R. H.: Accoutumance croisée de E. coli à Гégard des antibiotiques .... 954

Regamey, R. H. und Bertschmann, M.:

Die Prüfung der Pertussis-Impfstoffe am Meerschweinchen .... 946

Regamey, R.A.: vide Hiltbold, B.

Rene, L.: vide Bonfils, S.

Richer, CL: vide Bonfils, S.

Richir, CL: vide Lambling, A.

Rivkine, A.: Fixation d'anions tensio-actifs sur des germes modifies par un traite ment physique, chimique ou enzymatique 1075

Rogala, D.: vide Milgrom, F.

Rychlo, A.: Kontrastdarstellung der Rickettsien in histologischen Schnitten mit

Giemsa-Farbstoff $\quad 858$

Rymkiewicz, D.: vide Meisel, $\mathrm{H}$.

Schade, R.O.K.: The Morbid Anatomy of Peptic Ulceration .

372

Schade, R. 0. K.: Non-Ulcer Dyspepsia and Gastric Cytology. (A preliminary report) . 608

Schlegel-Opbecht, E.:

Reversion von A minus- zu A plus-Mutanten bei Colimastitisstämmen 994

Schobnagel, H. E.: vide Straub, M.

Secrétan, Ph.: vide Demole, M.

Shehadi, W. H.: Radiological and Geographical Considerations in the Diagnosis of

Peptic Ulcer 345

Simson, I.: vide Higginson, J.

Skurski, A.: Mechanism of the Opsonocytophagic Test in Brucellosis

Skurski, A.: vide Kañtoch, M.

Skubski, A.: vide Lisowski, J.

Slopek, St. and Dabbowski, L.:

Characteristics of the New Thermo-Labile Antigens Occurring in

Enterobacteriaceae 1037

Slopek, St. and Metzgeb, M.:

Serological Studies on Shigella Strains Isolated in Poland during

1953-1956 32

Sotgiu, G. et Pisi, E.:

Aspects cliniques de la pathologie géographique de Гulcère gastro-

duodénal 260

Spiba, J. J.: A Common Food Factor as the Etiological Element in the Geograph

ical Distribution of Chronic Peptic Ulcer

500 
Stebzl, J.: vide Trnka, Z.

Stbaub, M. and Schobnagel, H.E.:

General Aetiology of Gastro-Duodenal Ulcer

242

Tibunarayanan, M. O., Vischer, W. A. und Bruhin, H.:

Untersuchungen über den Wirkungsmechanismus der Thiosemicar-

bazone 964

Thomas, J.: vide Delarue, J.

Tokoro, Y.: The Lesions in other Organs in the Course of Gastroduodenal Ulcers

(Correlation of Gastroduodenal Ulcers Based upon a Histopatholo-

gical Evaluation of Background Organs) 504

Tomcsik, J. und Baumann-Grace, J.B.:

Sporulation und spezifische Sporangium-Reaktion 914

Tomcsik, J.: vide Baumann-Grace, J.B.

Trnka, Z., Málek, P., Stebzl, J. and Kolc, J.:

Experimental Contributions to the Lymphatic - Pathogenesis of An

thrax Infection 1082

VanËk, J.: Vergiftung mit Kreuzkraut (Senecio) als Ursache der Zdárer Pferde-

seuche 821

Velicangil, S.: vide Eser, S.

Viola, G.: vide Mabiani, P. L.

Virtanen, A.I.: Antimikrobiologische Substanzen in unseren Kulturpflanzen und ihre

Bedeutung für die Pflanzen und für die Ernährung des Menschen und

der Tiere $\quad 970$

Vischer, W.A.: vide Tirunabayanan, M.0. Vogel, A.: vide Lindenmann, J. Walczak, W.: vide

Pakula, R.

Watkinson, G.: The Autopsy Incidence of Chronic Peptic Ulceration, a National and

Regional Survey of 20000 Examinations Performed in Leeds, England,

between 1930 and 1949 and in 9 towns in England and Scotland in

1956405

Watt, J.: Acid Gastric Juice and Focal Ischaemia in the Pathogenesis of Mul

tiple Acute Ulceration 322

Weinbach, R.: Die Verwendbarkeit formolbehandelter Erythrocyten als Antigen-

träger in der indirekten Haemagglutination. I. Mitteilung 1043

Welsch, M.: Formation de protoplastes d'Escherichia coli sous $\Gamma$ influence de la

glycine et d'autres acides amines 741

Went, L.N. and MacIver, J.E.:

Distribution of Abnormal Haemoglobins in Jamaica 614

Wicher, K.: vide Milgrom, F.

Wieczorek, Z.: vide Kañtoch, M.

Wieczorek, Z.: vide Lisowski, J.

Wiesmann, E.: Serologische Befunde bei Adeno-Virus-Infektionen, Ornithose und Lues. (Vorläufige Mitteilung) 935

Windisch, F.: Endergonische Enzymreaktionen in Abhängigkeit vom anoxygenen

Energiepotential 1052

Wyler, R. und Kradolfer, F.: 
Ausdruck unspezifischer Resistenzverschiebung im Virustiter infizierter Mäuseorgane 1018

Wyler, R.: vide Kradolfer, F.

Zakrzewski, K.: vide Pakula, R.

Zbinden, G. und Pletscher, A.:

Experimentelle Untersuchungen über 5-Hydroxytryptamin-Gehalt und enterochromaffine Zellen bei chronischer Reizung des Magen-

Darm-Traktes durch Aethylalkohol 1137

Gesellschaftsberichte - Society Transactions - Sociétés

Verhandlungen der 6. Konferenz der Internationalen Gesellschaft für Geographische Pathologie

- Comptes Rendus de la 6e Conference de la Société Internationale de Pathologie Géographique

- Transactions of the 6th Meeting of the International Society of Geographical

Pathology, Paris, 9.-11. Juli 1957

Statute de la Société Internationale de Pathologie Géographique . . 635

Statutes of the International Society of Geographical Pathology . 637

Statuten der Internationalen Gesellschaft für Geographische Patho

logie 640

Schweizerische Mikrobiologische Gesellschaft. 17. Jahresversammlung,

28. und 29. Juni 1958, in Rheinfelden. - Société Suisse de Microbio-

logie. 17e assemblée annuelle, les 28 et 29 juin 1958, à Rheinfelden 901

Mitgliederliste der Schweizerischen Mikrobiologischen Gesellschaft. -

Membres de la Société Suisse de Microbiologie 1026

Buchbesprechungen - Book Reviews - Livres Nouveaux . 119,643,769,900,1032,1144

Varia 1036

Register rerum Vol. $21 \quad 1149$

Register nominum Vol. $21 \quad 1170$

Alle Rechte, insbesondere das der Übersetzung in fremde Sprachen, vorbehalten Ohne

ausdrückliche Genehmigung des Verlages ist es auch nicht gestattet, dieses Buch Oder * Teile

daraus auf photomechanisehem Wege (Photokopíe, Mikrokopie) zu vervielfältigen.

(C)

Copyright 1958 by S. Karger AG., Basel Printed in Switzerland by Buchdruckerei Friedrich

Reinhardt AG., Basel Cliches: Aberegg-Steiner \& Cie. AG., Bern, und Sleiner \& Cie. AG., Basel 